Drift stabilization of ballooning modes in a high- $\langle\beta\rangle$ LHD configuration

This article has been downloaded from IOPscience. Please scroll down to see the full text article.

2010 Nucl. Fusion 50025009

(http://iopscience.iop.org/0029-5515/50/2/025009)

View the table of contents for this issue, or go to the journal homepage for more

Download details:

IP Address: 128.178.50.70

The article was downloaded on 19/07/2010 at 16:01

Please note that terms and conditions apply. 


\title{
Drift stabilization of ballooning modes in a high- $\langle\beta\rangle$ LHD configuration
}

\author{
L. Brocher ${ }^{1}$, W.A. Cooper ${ }^{1}$, J.P. Graves ${ }^{1}$, G.A. Cooper $^{2}$, \\ Y. Narushima ${ }^{3}$ and K.Y. Watanabe ${ }^{3}$ \\ ${ }^{1}$ Ecole Polytechnique Fédérale de Lausanne, Centre de Recherches en Physique des Plasmas, \\ Association Euratom-Suisse, CH-1015 Lausanne, Switzerland \\ ${ }^{2}$ Physics Department, University of the South, Sewanee, TN 37383, USA \\ ${ }^{3}$ National Institute for Fusion Science, Oroshi-cho 322-6, Toki 509-5292, Japan \\ E-mail: lucie.brocher@epfl.ch
}

Received 1 September 2009, accepted for publication 7 December 2009

Published 15 January 2010

Online at stacks.iop.org/NF/50/025009

\begin{abstract}
Ideal MHD yields at best inconclusive predictions about the stability of the LHD heliotron for $\langle\beta\rangle \geqslant 3 \%$. We investigate the impact of the drift stabilization of ballooning modes for the inward-shifted LHD configuration (vacuum magnetic axis $R_{0} \sim 3.5 \mathrm{~m}$ ). The background equilibrium is considered anisotropic in which the neutral beam ions contribute about $1 / 4$ fraction of the total diamagnetic beta, $\left\langle\beta_{\text {dia }}\right\rangle$. A drift corrected ballooning mode equation obtained from the linearized gyrokinetic equation is expanded assuming that the hot particle drifts are much larger than the mode frequency. The fast particle pressure gradients contribute weakly to both the instability drive and the diamagnetic drift stabilization (which is dominated by the thermal ion diamagnetic drifts) for $\left\langle\beta_{\text {dia }}\right\rangle \in[0,4.8] \%$. In the single-fluid limit (diamagnetic drifts ignored), the thermal pressure gradients drive ballooning modes in a broad region encompassing the outer $60-90 \%$ of the plasma volume at $\left\langle\beta_{\text {dia }}\right\rangle \approx 4.8 \%$. To stabilize these modes, we find that diamagnetic drift corrections must be invoked (mainly due to the thermal ions). The energetic ion diamagnetic drifts play a role only for low wave number values, $k_{\alpha} \leqslant 8$. It has been verified that the fast particle drift ordering imposed by the model is amply satisfied for on-axis hot particle to thermal density $N_{\mathrm{h} 0} / N_{\mathrm{i} 0} \approx 1 \%$ even at high $\left\langle\beta_{\text {dia }}\right\rangle$.
\end{abstract}

PACS numbers: 52.35.Py

(Some figures in this article are in colour only in the electronic version)

\section{Introduction}

In the LHD device, high- $\langle\beta\rangle(\langle\beta\rangle \simeq 5 \%)$ discharges are obtained with an inward-shifted configuration, low density $\left(<3 \times 10^{-19} \mathrm{~m}^{-3}\right)$, strong tangential neutral beam injection $(\approx 14 \mathrm{MW}, \approx 150-180 \mathrm{keV})$ [1]. At low densities, the pressure anisotropy driven by the neutral beam ions can be very significant with $p_{\|}>p_{\perp}$ [2]. The ideal MHD model predicts ballooning instability in heliotron on devices well below the experimentally achieved $\langle\beta\rangle$ values on LHD [3]. One very interesting observation that has been made shows that the LHD experimental data points [4] align with the theoretically calculated ideal MHD stability diagrams for global $n=1$ modes when the hot particle contributions to the pressure gradients are neglected. This tends to suggest that extensions to the ideal MHD model, like that from the Kruskal-Oberman energy principle [5] or even more appropriately the Astronmotivated energy principle developed by Johnson et al [6] may be more relevant for the accurate modelling of the stability properties of the LHD device at high- $\langle\beta\rangle$. However, using these models the analysis of global modes and the Mercier criterion predict instability (albeit weakly with the rigid hot particle model of Johnson et al) for $\langle\beta\rangle \approx 4 \%$ and lower [7, 8].

The ballooning criteria are particularly severe for field lines that cross the regions with most destabilizing magnetic field line curvature. Finite radial corrections require the evaluation of ballooning stability on all field lines and all radial wave numbers. For pressure profiles that are more peaked than in the experiment, the unstable domains are topologically spherical [3,9] and global structures are difficult to construct leading to inconclusive stability predictions. For profiles that are box-like (broader that in the experiment), the ballooning unstable domains are topologically cylindrical from which unstable domains can be computed [10].

As the fluid models predict instability in the LHD device, we need to invoke kinetic effects to verify whether the theory can be reconciled with the experimental observations of stable operation with range $0<\left\langle\beta_{\mathrm{dia}}\right\rangle<5 \%$, where $\left\langle\beta_{\mathrm{dia}}\right\rangle$ is the 
volume averaged diamagnetic component of $\langle\beta\rangle$. Diamagnetic drift corrections to the ballooning mode equation, as applied to tokamak devices, have been explored $[11,12]$. In the LHD device, diamagnetic drift corrections have been evaluated for global modes [13]. At high $\langle\beta\rangle \approx 4 \%$, if plasma compression effects are included, it is speculated that the growth rates are sufficiently small to be stabilized by the diamagnetic drifts. However, at lower $\langle\beta\rangle \approx 1 \%$, the modes growth rates are too large for drift stabilization, therefore the question of accessibility to high $\langle\beta\rangle$ remains unanswered.

A drift-magnetohydrodynamic model, where the fast particle drifts are much larger than the typical mode frequency/growth rate in the ballooning limit, has been previously derived for the case of energetic ions like those from neutral beam injection [14]. A diamagnetic drift corrected ballooning mode equation is derived. It is worthwhile to note that with energetic electrons rather than ions, a virtually identical equation is obtained, although the intermediate steps in the derivation are different due to the different orderings that must be applied [15]. This ballooning equation is in many aspects a diamagnetic drift extension of the fluid model of Johnson et al [6], where the fast particles constitute a rigid non-interacting population.

In this work, we shall only explore the most unstable field lines on each flux surface. A diamagnetic drift ballooning model that addresses radial corrections has been developed to connect ballooning structures to global modes by ray tracing techniques in the ballooning space [16]. It is useful as it can be used to determine the wave number $k_{\alpha}$ which is merely a parameter in the asymptotic ballooning limit. In section 2 we present the MHD equilibrium state that has been used for anisotropic pressure profiles. A bi-Maxwellian pressure distribution function has been considered to model fast particle species. The single-fluid ballooning and the driftMHD equation, in their variational form, are developed and solved in section 3 using a COOL finite element discretization scheme and an inverse vector iteration technique [17]. A selection of numerical results is shown in section 4. Finally a summary and a conclusion end the paper.

\section{The MHD equilibrium state}

Let us consider an anisotropic pressure background equilibrium in which $\mathrm{H}$-beam ions are injected and contribute about a quarter fraction of the total diamagnetic beta.

The equilibrium state for anisotropic pressure equilibria is computed with the ANIMEC code [18] to model the inwardshifted LHD configuration at high- $\langle\beta\rangle$. To model the threedimensional equilibrium we choose to consider an anisotropic pressure bi-Maxwellian distribution function model to describe the fast particle species [19]. This model allows energetic particle deposition and its corresponding contribution to the parallel and perpendicular pressure to be localized in the central region of the plasma or off-axis either on the high or low field side through the choice of value assigned to a critical magnetic field strength $B_{\mathrm{c}}$ together with a hot particle temperature profile amplitude factor $T_{\|} / T_{\perp}$. This type of distribution function closely approximates the solution of a Fokker-Planck equation, particularly when ion cyclotron resonance heating is applied [20, 21].

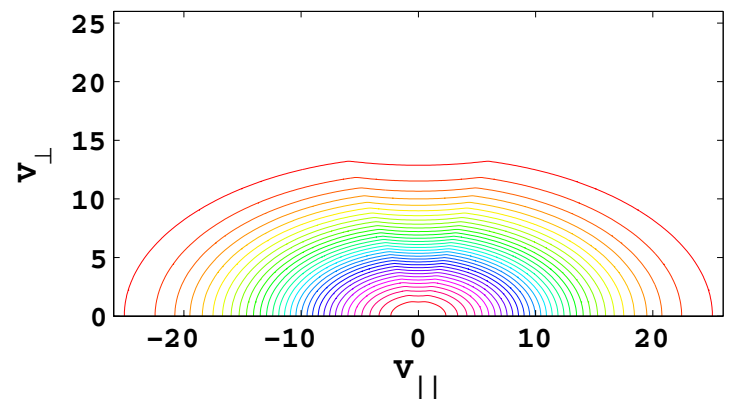

Figure 1. Contour of a bi-Maxwellian distribution function in velocity space at $B_{\mathrm{c}} / B=1.25$ with $T_{\|} / T_{\perp}=3$. A loss-cone feature is evident for small $v_{\perp}$.

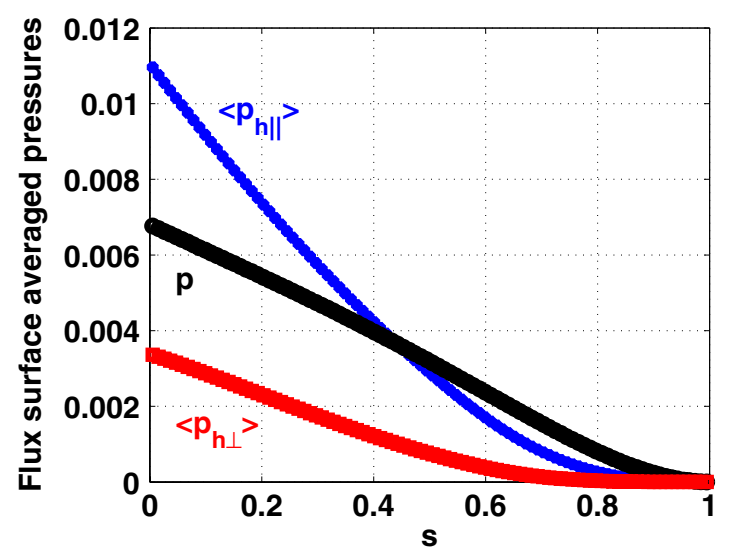

Figure 2. Radial profiles of the total, perpendicular and parallel pressure at $\left\langle\beta_{\text {dia }}\right\rangle=4.79 \%$.

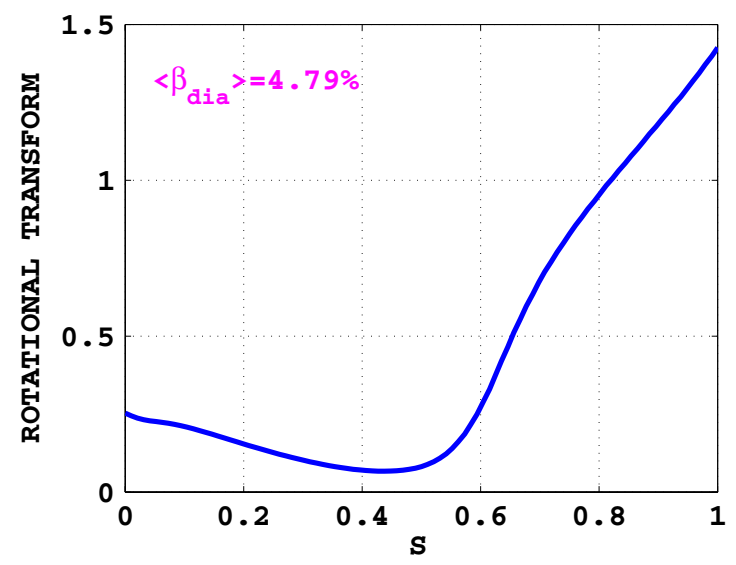

Figure 3. The rotational transform profile as a function of the normalized toroidal flux $s$ at $\left\langle\beta_{\text {dia }}\right\rangle=4.79 \%$.

Figure 1 presents contours of the bi-Maxwellian distribution function in velocity space with $B_{\mathrm{c}} / B=1.25$ and $T_{\|} / T_{\perp}=3$. Figures 2 and 3 illustrate profiles of rotational transform and pressure as a function of the magnetic normalized toroidal flux $s$, for the configuration with $\left\langle\beta_{\mathrm{dia}}\right\rangle=$ $4.79 \%$. Here and elsewhere, the definitions of $\left\langle\beta_{\|}\right\rangle$and $\left\langle\beta_{\perp}\right\rangle$ involve total $p_{\| \mid}$and $p_{\perp}\left(p_{\|}=p+p_{|| \mathrm{h}}\right)$, where $p$ identifies the thermal pressure. We particularly choose to take a heliotron system with a large parallel anisotropy $\left(p_{\|}>p_{\perp}\right)$. The input profiles used and the parameters for the three-dimensional equilibrium will be presented in the next section. 


\subsection{Input profiles and parameters}

The hot particle deposition layer is concentrated around a magnetic field value $B_{\mathrm{C}}=0.425 \mathrm{~T}$. The profiles required for the equilibrium calculation are

- Thermal pressure: $p(s)=p(0)(1-s)\left(1-s^{4}\right)$.

- Hot particle pressure amplitude: $p_{\mathrm{h}}(s)=p_{\mathcal{H}}(1-s)$.

- Anisotropy factor: $\left[T_{\perp} / T_{\|}\right](s)=\left[T_{\perp} / T_{\|}\right](0)\left(1-s^{2}\right)$.

- Toroidal current: $2 \pi J(s)=0$.

- Radial variable: $0 \leqslant s \leqslant 1(\propto$ enclosed toroidal magnetic flux).

- $p(0)$ and $p_{\mathcal{H}}$ are chosen so that $\left\langle\beta_{\mathrm{dia}}\right\rangle=\left\langle 2 \mu_{0} p_{\perp} / B^{2}\right\rangle \in$ $[0,4.79] \%$ and $\left\langle\beta_{\text {th }}\right\rangle=\left\langle 2 \mu_{0} p / B^{2}\right\rangle \in[0.3,3.3] \%$ (thus hot and thermal pressures are increased at the same rate).

- $\left[T_{\perp} / T_{\|}\right](0)=1 / 3$ yields $\left\langle\beta_{\|}\right\rangle /\left\langle\beta_{\text {dia }}\right\rangle \simeq 1.62$.

The maximum value experimentally achieved [1] is $\left\langle\beta_{\mathrm{dia}}\right\rangle \simeq$ $4.8 \%$.

In anisotropic pressure plasmas there is not a unique definition of the parameter $\langle\beta\rangle$. One choice is $\left\langle\beta_{\text {dia }}\right\rangle$, which experimentally is the easiest to measure. But there are other possibilities that can be considered. Taking $\langle\beta\rangle=$ $\left\langle 2 \mu_{0} W / B^{2}\right\rangle$, we could examine $W=\left(2 p_{\perp}+p_{\|}\right) / 3$ as an option, because there are two degrees of freedom in the perpendicular direction and only one parallel to the magnetic field lines. In this case, for $\left\langle\beta_{\mathrm{dia}}\right\rangle \simeq 4.8 \%$, LHD would achieve $\langle\beta\rangle \simeq 5.79 \%$. From the perspective of MHD stability, the driving terms for pressure driven modes involve the sum $W=p_{\perp}+p_{\|}$. In this case we can define an equal weight $\left\langle\beta_{\mathrm{EW}}\right\rangle$ which reaches $6.28 \%$.

The large parallel anisotropy associated with the tangential neutral beam system in the LHD implies that the 'MHD stability relevant' $\langle\beta\rangle$ value, in this device, may well exceed the experimentally reported $\left\langle\beta_{\text {dia }}\right\rangle \simeq 5 \%$.

\section{Drift-MHD ballooning stability equation}

As a starting point, we have examined the linearized gyrokinetic equation in ballooning space [22], in a configuration where the drift effects of energetic and thermal species play a role and where trapped particle effects are neglected. A comprehensive treatment is required to investigate the impact of trapped particles and other kinetic effects in stellarator geometry with models like the FULL code [23]. The kinetic modifications we include are limited to diamagnetic drift extensions to the fluid MHD model. We concentrate thus only on instabilities that are directly linked to the MHD branch. The critical approximation involved for this equation is $\omega / \omega_{\mathrm{dh}} \ll 1$ (a more detailed discussion of $\omega_{\mathrm{dh}}$ shall be examined in the next section), which in its variational form [14] reads as

$$
\begin{aligned}
\int_{-\infty}^{\infty} \mathrm{d} \theta & \left\{\frac{\sigma k_{\perp}^{2}}{\sqrt{g} B^{2}}|\sqrt{g} \boldsymbol{B} \cdot \nabla \chi|^{2}-\frac{k_{\alpha} \sqrt{g} p^{\prime}(s)}{\psi^{\prime}(s) B^{2}}\right. \\
\cdot & {\left.\left[\left(1+\frac{\sigma}{\tau}\right) \boldsymbol{B} \times \boldsymbol{k}_{\perp} \cdot \boldsymbol{\kappa}-\left.\frac{k_{\alpha}}{\psi^{\prime}(s)} \frac{\partial p_{\perp \mathrm{h}}}{\partial s}\right|_{B}\right]|\chi|^{2}\right\} } \\
= & \int_{-\infty}^{\infty} \mathrm{d} \theta M_{\mathrm{i}} N_{\mathrm{i}} \frac{\sqrt{g} k_{\perp}^{2}}{B^{2}} \omega\left[\omega-\left(\omega_{* \mathrm{pi}}+\frac{M_{\mathrm{h}} N_{\mathrm{h}} \omega_{* \mathrm{~h}}}{M_{\mathrm{i}} N_{\mathrm{i}} b_{\mathrm{h}}}\right)\right]|\chi|^{2}
\end{aligned}
$$

In the derivation of equation (1), we have applied the covering space concept to reconcile mode structure periodicity in a toroidal system with finite magnetic shear [24]. Thus, in ballooning space, each field line labelled by $\alpha$ can be uniquely identified. The radial wave number is $\theta_{k} \equiv$ $k_{q} / k_{\alpha}$, the wave vector is $k_{\perp}=k_{\alpha}[\nabla \phi-q(s) \nabla \theta-$ $\left.q^{\prime}(s)\left(\theta-\theta_{k}\right) \nabla s\right]$ [24]. Note that in axisymmetric tokamaks, the wave number $k_{\alpha}$ corresponds to the toroidal mode number $n$. The firehose stability parameter is $\sigma=\left(1 / \mu_{0}\right)-$ $\left.(1 / B)\left(\partial p_{\|} / \partial B\right)\right|_{s}$, the mirror stability parameter is $\tau=$ $\left(1 / \mu_{0}\right)+\left.(1 / B)\left(\partial p_{\perp} / \partial B\right)\right|_{s}$, the thermal ion diamagnetic drift frequency is $\omega_{* \mathrm{pi}}=-k_{\alpha} p^{\prime}(s) /\left(2 Z_{\mathrm{i}} e \psi^{\prime}(s) N_{\mathrm{i}}\right)$, the hot particle Larmor radius term is $b_{\mathrm{h}}=k_{\perp}^{2} \rho_{\mathrm{h}}^{2}=k_{\perp}^{2} p_{\perp \mathrm{h}} M_{\mathrm{h}} /\left(Z_{\mathrm{h}}^{2} e^{2} B^{2} N_{\mathrm{h}}\right)$, the hot ion diamagnetic drift frequency is given by $\omega_{* \mathrm{~h}}=-\left.\left[k_{\alpha} p_{\perp \mathrm{h}} /\left(Z_{\mathrm{h}} e \psi^{\prime}(s) N_{\mathrm{h}}^{2}\right)\right] \frac{\partial N_{\mathrm{h}}}{\partial s}\right|_{B}$, the hot particle density derived from the Bi-Maxwellian distribution is given by $N_{\mathrm{h}}(s, B)=N_{\mathrm{h}}\left(s, B_{\mathrm{C}}\right) \mathcal{C}(s, B)$ :

- For $B \geqslant B_{\mathrm{C}}$

$$
\mathcal{C}(s, B)=\frac{\frac{B}{B_{\mathrm{C}}}}{1-\frac{T_{\perp}}{T_{\|}}\left(1-\frac{B}{B_{\mathrm{C}}}\right)}
$$

- For $B<B_{\mathrm{C}}$

$$
\begin{aligned}
\mathcal{C}(s, B)= & \frac{\frac{B}{B_{\mathrm{C}}}}{1-\frac{T_{\perp}}{T_{\|}}\left(1-\frac{B}{B_{\mathrm{C}}}\right)} \\
& \times\left[1-\frac{2\left(\frac{T_{\perp}}{T_{\|}}\right)^{3 / 2}\left(1-\frac{B}{B_{\mathrm{C}}}\right)^{3 / 2}}{1+\frac{T_{\perp}}{T_{\|}}\left(1-\frac{B}{B_{\mathrm{C}}}\right)}\right] .
\end{aligned}
$$

The hot particle density profile is chosen as $N_{\mathrm{h}}\left(s, B_{\mathrm{C}}\right)=$ $N_{\mathrm{h} 0}(1-s)^{2}$. The particular critical assumption $\omega / \omega_{\mathrm{dh}} \ll 1$ implies that hot particle species are taken in such a way that they traverse unstable regions too quickly to significantly contribute to the instability drive. Consequently, the thermal ion and electron pressure gradients can dominate the ballooning stability properties. Conversely, the term that involves the hot particle diamagnetic drift stabilization $\omega_{* \mathrm{~h}}$ has a correction proportional to $\rho_{\mathrm{h}}^{-2}$ which shows that the larger the hot particle Larmor radius, the smaller the stabilization effect.

\subsection{The single-fluid ballooning limit and the solution method}

As a first step to solve the drift-MHD equation, the TERPSICHORE code is used to transform the equilibrium state previously computed with the help of ANIMEC to Boozer magnetic coordinates [25].

To solve the resulting equation we explore the single-fluid ballooning limit obtained by imposing $\omega_{* \mathrm{pi}}=\omega_{* \mathrm{~h}}=0$. The 
equation reduces to

$$
\begin{aligned}
\int_{-\infty}^{\infty} \mathrm{d} \theta & \left\{\frac{\sigma k_{\perp}^{2}}{\sqrt{g} B^{2}}|\sqrt{g} \boldsymbol{B} \cdot \nabla \chi|^{2}-\frac{k_{\alpha} \sqrt{g} p^{\prime}(s)}{\psi^{\prime}(s) B^{2}}\right. \\
\cdot & {\left.\left[\left(1+\frac{\sigma}{\tau}\right) \boldsymbol{B} \times \boldsymbol{k}_{\perp} \cdot \boldsymbol{\kappa}-\left.\frac{k_{\alpha}}{\psi^{\prime}(s)} \frac{\partial p_{\perp \mathrm{h}}}{\partial s}\right|_{B}\right]|\chi|^{2}\right\} } \\
= & -\gamma_{\mathrm{F}}^{2} \int_{-\infty}^{\infty} \mathrm{d} \theta M_{\mathrm{i}} N_{\mathrm{i}} \frac{\sqrt{g} k_{\perp}^{2}}{B^{2}}|\chi|^{2} .
\end{aligned}
$$

Note that if $p_{\perp \mathrm{h}}=0$ then $\sigma / \tau=1$, which recovers the incompressible ideal MHD model. The eigenfunction $\chi$ is discretized using COOL finite elements, which are based on variable-order Legendre polynomials. The variational problem is then reduced to a special block-pentadiagonal matrix eigenvalue equation:

$$
\mathcal{A} Y=\lambda \mathcal{B} Y
$$

where the eigenvalue is $\lambda=-\gamma_{\mathrm{F}}^{2}$. The matrix eigenvalue equation is solved using an inverse vector iteration technique with the BECOOL code [17], and the order of the polynomial chosen is typically cubic. We are particularly interested in the field lines that cross the most destabilizing curvature region on each flux surface; therefore, the fluid line label $\alpha=0$ is imposed. We also choose $\theta_{k}=0$.

\subsection{Ballooning study with diamagnetic drift corrections}

After solving the single-fluid ballooning limit, we finally apply a perturbative approach to solve the drift-MHD ballooning equation. The eigenfunction $\chi$ from the single-fluid ballooning model is taken as a test eigenfunction. The resulting dispersion relation is derived from equation (1):

$$
\omega^{2}-\omega\left(\omega_{* \mathrm{pi}}+\omega_{* \mathrm{heff}}\right)+\gamma_{\mathrm{F}}^{2}=0
$$

where $\omega_{* \text { heff }}$ is the effective hot particle diamagnetic drift frequency:

$$
\begin{aligned}
\omega_{* \text { heff }} & =\left\langle\frac{M_{\mathrm{h}} \omega_{* \mathrm{~h}}}{M_{\mathrm{i}} N_{\mathrm{i}} b_{\mathrm{h}}}\right\rangle \\
& =\frac{\int_{-\infty}^{\infty} \mathrm{d} \theta \frac{\sqrt{g}}{\psi^{\prime}(s)}\left(\frac{k_{\perp}^{2}}{B^{2}}\right)\left(\frac{M_{\mathrm{h}} N_{\mathrm{h}} \omega_{* \mathrm{~h}}}{M_{\mathrm{i}} N_{\mathrm{i}} b_{\mathrm{h}}}\right)|\chi|^{2}}{\int_{-\infty}^{\infty} \mathrm{d} \theta \frac{\sqrt{g}}{\psi^{\prime}(s)}\left(\frac{k_{\perp}^{2}}{B^{2}}\right)|\chi|^{2}} .
\end{aligned}
$$

The integrals in the numerator and the denominator are performed with the same Gauss-Legendre quadrature rule that is applied in the BECOOL code. All frequencies (and growth rate) are normalized to the toroidal Alfvén frequency $\omega_{\mathrm{A}}=v_{\mathrm{A}} / R_{0}$, where $v_{\mathrm{A}}=B_{0} / \sqrt{\mu_{0} M_{\mathrm{i}} N_{\mathrm{io}}}$.

From the dispersion relation (equation (4)) we can assert that if $\left|\omega_{* \text { heff }}+\omega_{* \mathrm{pi}}\right| / 2 \geqslant \gamma_{\mathrm{F}}$, the mode growth rate is equal to zero and the mode is stabilized; conversely, if $\mid \omega_{* \text { heff }}+$ $\omega_{* \mathrm{pi}} \mid / 2<\gamma_{\mathrm{F}}$ the resulting frequency $\omega$ is complex and the mode remains unstable.

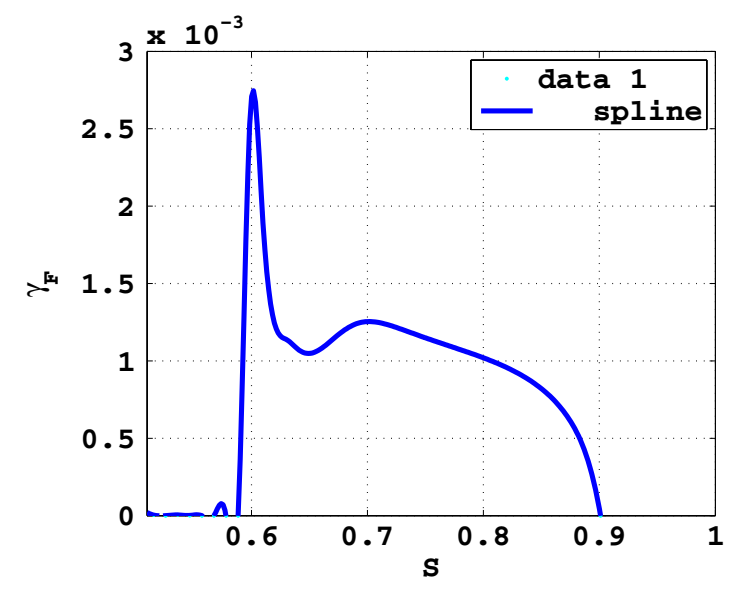

Figure 4. The fluid growth rate profile at $\left\langle\beta_{\text {dia }}\right\rangle=4.79 \%$ for field lines that cross the region where the magnetic curvature is most destabilizing in the LHD configuration investigated.

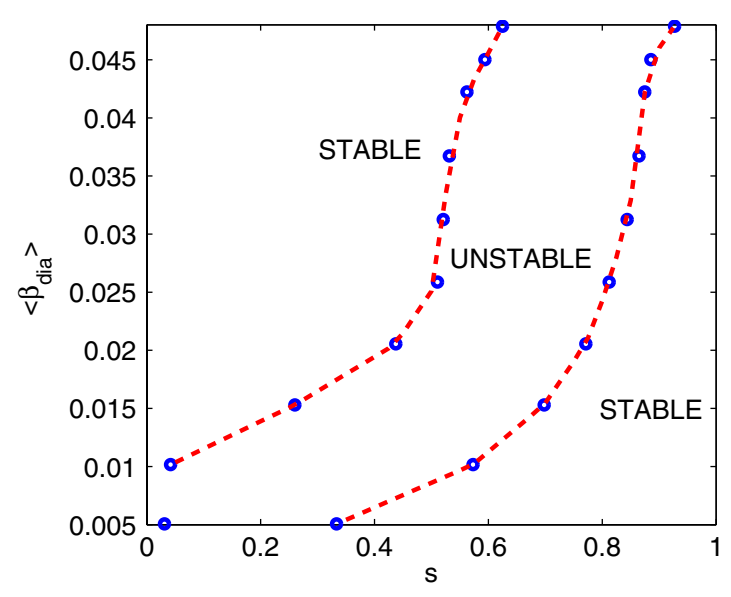

Figure 5. The ballooning unstable domain in a LHD configuration for field lines that cross the most destabilizing curvature region.

\section{Numerical results}

The aim of this section is to present our numerical results that include the modification of the ballooning mode equation including diamagnetic drifts effects. After the determination of the single-fluid unstable regions as a function of the normalized toroidal flux $s$, we apply the perturbative approach taking $\chi$ from the single-fluid ballooning model as a test eigenfunction and solve the quadratic dispersion relation.

The calculation of the growth rate $\gamma_{\mathrm{F}}$ at $\left\langle\beta_{\mathrm{dia}}\right\rangle=4.79 \%$ as a function of the radial variable (see figure 4 ) identifies which regions are destabilized by ballooning modes.

We focus on the most unstable surface which, for $\left\langle\beta_{\text {dia }}\right\rangle=$ $4.79 \%$, corresponds to $s=0.599$ as shown in figure 4 . For field lines that cross the most destabilizing curvature region in the range $\left\langle\beta_{\text {dia }}\right\rangle \in[0,4.79] \%$, we determine the unstable band (see figure 5), according to the single-fluid model. This ballooning unstable band moves from the plasma core, at low $\left\langle\beta_{\text {dia }}\right\rangle$, to the plasma boundary, at $\left\langle\beta_{\text {dia }}\right\rangle \backsim 5 \%$. We now apply our drift-magnetohydrodynamic ballooning mode equation in order to investigate the impact of thermal ion and effective hot particle diamagnetic drifts. All simulations were computed for

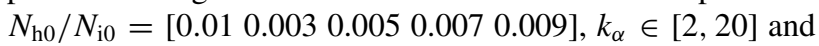



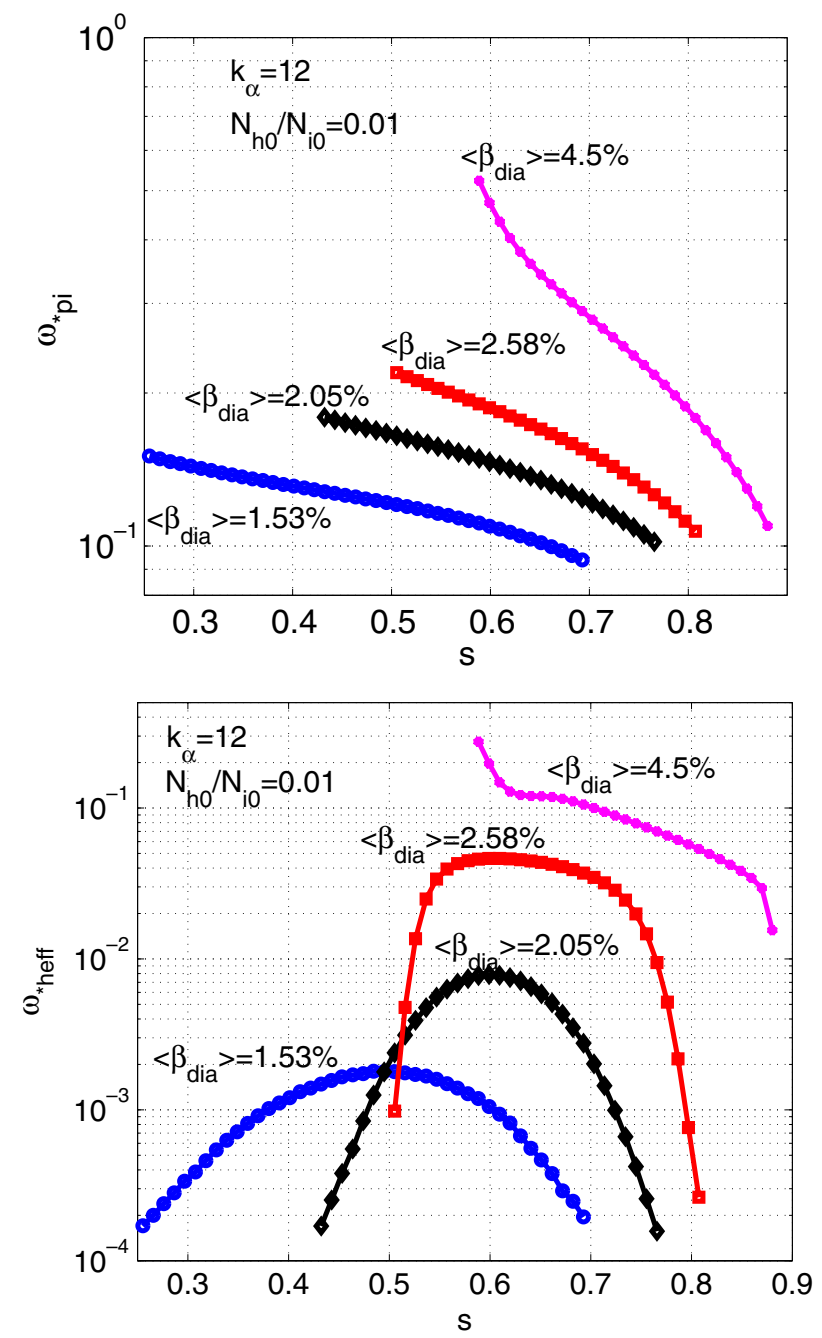

Figure 6. The thermal ion diamagnetic $(a)$ and the effective hot particle $(b)$ diamagnetic drift frequencies for several values of $\left\langle\beta_{\mathrm{dia}}\right\rangle$ as a function of the radial position $\left(k_{\alpha}=12, N_{\mathrm{h} 0} / N_{\mathrm{i} 0}=0.01\right)$. The $\omega_{* \mathrm{pi}}$ are calculated only within the unstable fluid frequency $\gamma_{\mathrm{F}}$ domain.

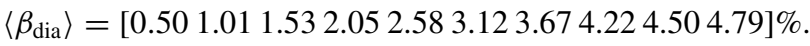
Only some results will be shown as an illustration.

In figure 6 we present the thermal ion diamagnetic $(a)$ and the effective hot particle $(b)$ diamagnetic drifts as a function of the radial position which corresponds to the unstable fluid domain. We have decided to show here the particular configuration with $k_{\alpha}=12$ and $N_{\mathrm{h} 0} / N_{\mathrm{i} 0}=0.01$ and for some values of $\left\langle\beta_{\text {dia }}\right\rangle$.

The impact of the thermal ion diamagnetic drift on plasma stability is more relevant than that of the effective hot particle diamagnetic drift (from 1 to 3 orders of magnitude larger). We note that, as $\left\langle\beta_{\text {dia }}\right\rangle$ becomes larger, the magnitude of $\omega_{* \text { heff }}$ starts to approach that of $\omega_{* \text { pi }}$. Figure 7 plots the mode frequency $\omega$, the thermal ion diamagnetic drift frequency $\omega_{* \mathrm{pi}}$, the effective hot particle frequency $\omega_{* \text { heff }}$ and the fluid growth rate $\gamma_{F}$ as a function of the wave number $k_{\alpha}$ for the magnetic field line that crosses the most destabilizing magnetic curvature region $\left(\left\langle\beta_{\text {dia }}\right\rangle=1.53 \%, N_{\mathrm{h} 0} / N_{\mathrm{i} 0}=0.01, s=0.49\right)$. Here $\omega_{* \mathrm{pi}} \gg \omega_{* \text { heff }}$, except at a very small value of $k_{\alpha}$. Hence the mode frequency $\omega$ is virtually equivalent to the thermal

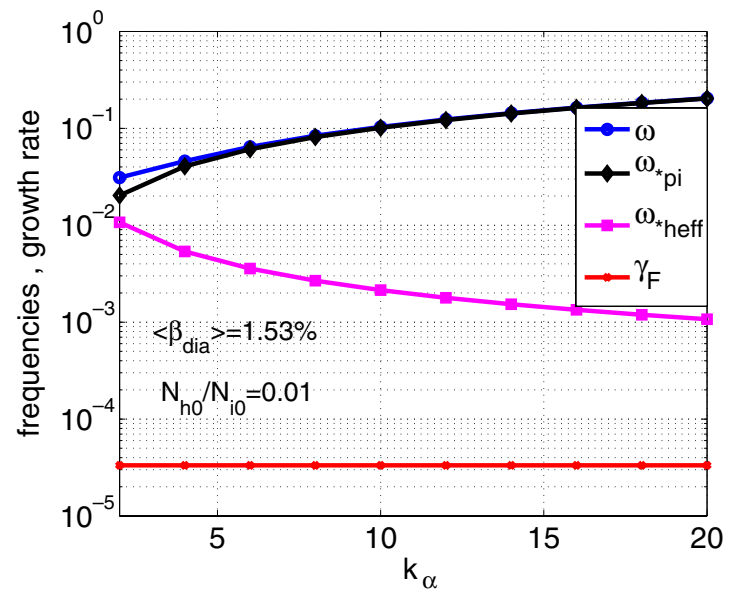

Figure 7. The mode frequency $\omega$ (blue circle $\bullet$ ), the thermal ion diamagnetic drift frequency $\omega_{* \text { pi }}$ (black diamond $\diamond$ ), the effective hot particle diamagnetic drift frequency $\omega_{* \text { heff }}$ (magenta square $\mathbf{\square}$ ) and the fluid growth rate $\gamma_{\mathrm{F}}$ (red cross $\times$ ) as a function of the wave number $k_{\alpha}$ for $\left\langle\beta_{\text {dia }}\right\rangle=1.53 \%$ and $N_{\mathrm{h} 0} / N_{\mathrm{i} 0}=0.01$ for $s=0.49$ (which corresponds to the most unstable field line).

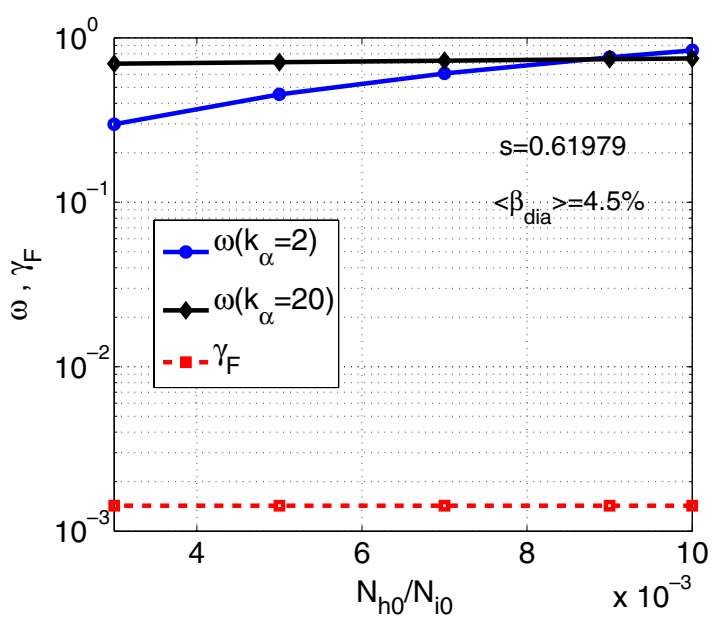

Figure 8. The mode frequency $\omega$ for $k_{\alpha}=2$ (blue circle $\bullet$ ) and $k_{\alpha}=20$ (black diamond $\diamond$ ) as a function of the hot particle to thermal density ratio for $\left\langle\beta_{\mathrm{dia}}\right\rangle=4.5 \%$ at the radial position $s=0.62$. For reference purposes, we also plot the fluid growth rate $\gamma_{\mathrm{F}}$ (which is by definition independent of $N_{\mathrm{h} 0} / N_{\mathrm{i} 0}$ ).

ion diamagnetic drift $\omega_{* \mathrm{pi}}$ because $\omega_{* \mathrm{pi}} \gg \gamma_{\mathrm{F}}$. Furthermore, because $\omega_{* \mathrm{pi}} \gg \gamma_{\mathrm{F}}$, the condition $\left|\omega_{* \text { heff }}+\omega_{* \mathrm{pi}}\right| / 2>\gamma_{\mathrm{F}}$ is satisfied, which implies that the complex mode frequency $\omega$ has a real part and the instability growth rate is zero.

Because the hot particle curvature and grad-B drifts are taken to be too large to contribute significantly to the instability, it would be consistent that the corresponding diamagnetic drift frequency should have a weak impact on the stability properties. That is exactly what happens in our case.

In figure 8 we see the mode frequency, for $\left\langle\beta_{\mathrm{dia}}\right\rangle=4.5 \%$, $k_{\alpha}=2$ and $k_{\alpha}=20$ as a function of the hot to thermal particle density ratio. For $k_{\alpha}=2$, the effective hot particle diamagnetic drift has an important contribution that scales as $N_{\mathrm{h} 0} / N_{\mathrm{i} 0}$ and consequently we see a corresponding increase in the frequency of the mode $\omega$. For $k_{\alpha}=20, \omega_{* \text { pi }} \gg \omega_{* \text { heff }}$, in this case $\omega$ is virtually independent of $N_{\mathrm{h} 0} / N_{\mathrm{i} 0}$. In figure 9 the normalized velocity-space averaged hot particle drift frequency is plotted 


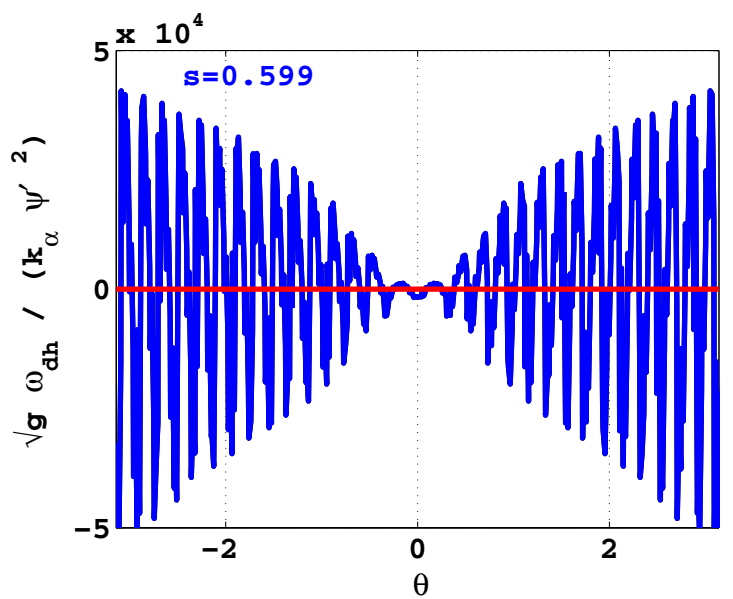

Figure 9. The normalized velocity-space averaged hot particle drift frequency as a function of the poloidal angle $\theta$ (in the range $-\pi<\theta<\pi$ ) on the most unstable field line that lies on the flux surface labelled with $s \approx 0.599$ at $\left\langle\beta_{\mathrm{dia}}\right\rangle \approx 4.79 \%$.

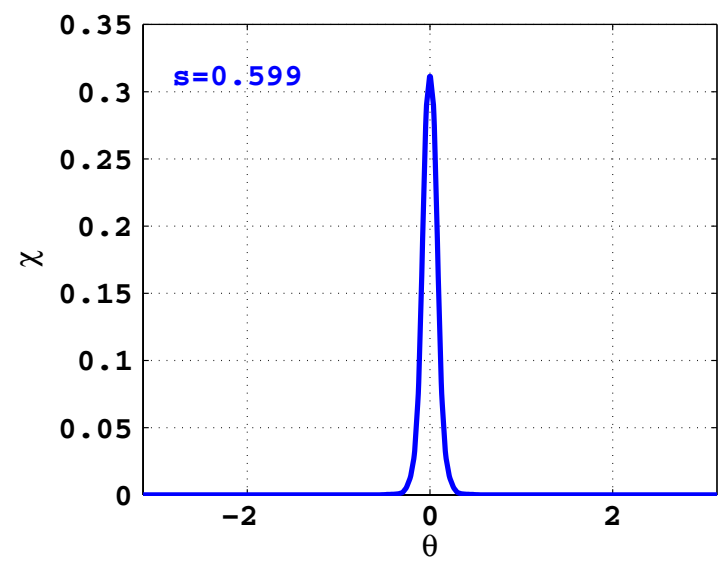

Figure 10. The fluid ballooning mode structure along the field line that crosses the most destabilizing curvature region in the LHD configuration on the surface $s=0.599$ at $\left\langle\beta_{\text {dia }}\right\rangle=4.79 \%$ (corresponding to $\gamma_{\mathrm{F}} \approx 2.75 \times 10^{-3}$ ).

as a function of the poloidal angle $\theta(\theta \in[-\pi, \pi])$, along the most unstable field line on the surface $s=0.599$ and for $\left\langle\beta_{\text {dia }}\right\rangle \approx 4.79 \%$. This frequency displays a strong oscillatory behaviour as expected. We, however, contend that its effect is most relevant only within the domain where the mode structure is finite and localized (see figure 10) (i.e. close to $\theta=0$ ).

We therefore define a mode-weighted total hot particle drift frequency (curvature + grad-B drift) as

$$
\begin{gathered}
\left\langle\omega_{\mathrm{dh}}\right\rangle=\frac{\int_{-\infty}^{\infty} \mathrm{d} \theta\left(\sqrt{g} / \psi^{\prime}\right) \omega_{\mathrm{dh}}|\chi|^{2}}{\int_{-\infty}^{\infty} \mathrm{d} \theta\left(\sqrt{g} / \psi^{\prime}\right)|\chi|^{2}}, \\
\omega_{\mathrm{dh}}=\frac{\int \mathrm{d}^{3} v F_{0 \mathrm{~h}} \hat{\omega}_{\mathrm{dh}}}{\int \mathrm{d}^{3} v F_{0 \mathrm{~h}}} .
\end{gathered}
$$

The single particle drift is defined as $\hat{\omega}_{\mathrm{d} j}=-\left(\omega_{\kappa} v_{\|}^{2}+\right.$ $\left.\omega_{B} v_{\perp}^{2} / 2\right) / \Omega_{j}$, where $\omega_{\kappa} \equiv \boldsymbol{B} \times \boldsymbol{k}_{\perp} \cdot \boldsymbol{\kappa} / \boldsymbol{B}, \omega_{B} \equiv \boldsymbol{B} \times \boldsymbol{k}_{\perp}$. $\boldsymbol{\nabla} \boldsymbol{B} / \boldsymbol{B}^{2}$ and $\Omega_{j}$ is the cyclotron frequency for species $j$. We need to compare mode and drift frequency at $\left\langle\beta_{\mathrm{dia}}\right\rangle=4.79 \%$.

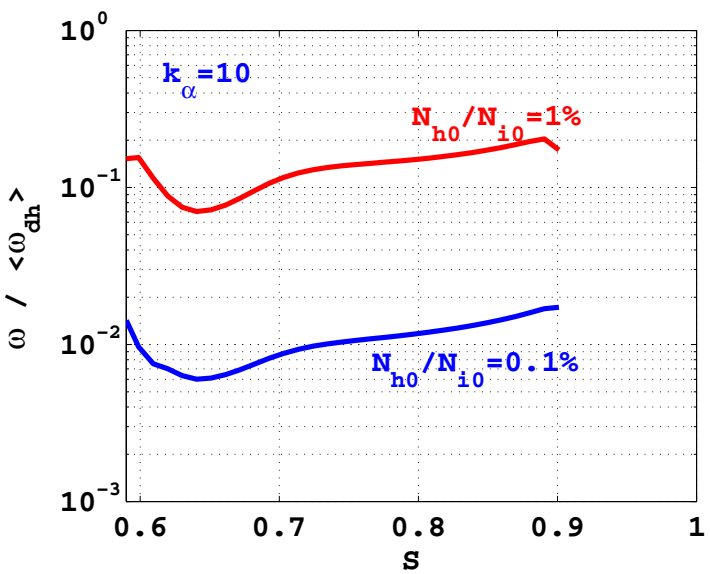

Figure 11. The ratio of mode frequency to total drift frequency (curvature $+\operatorname{grad} \mathrm{B}$ ) as a function of $s$ (in the range of unstable $\gamma_{\mathrm{F}}$ ) for $\left\langle\beta_{\mathrm{dia}}\right\rangle=4.79 \%, k_{\alpha}=10, N_{\mathrm{h} 0} / N_{\mathrm{i} 0}=0.01$ and 0.001 .

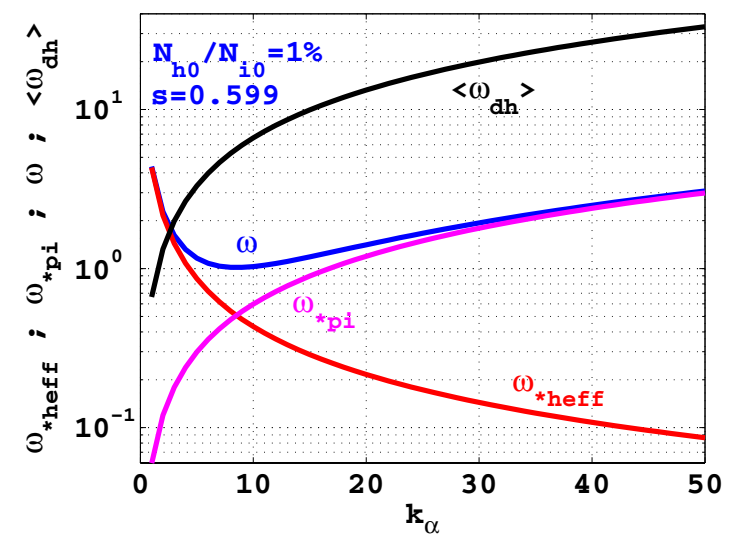

Figure 12. The scaling of total magnetic drift, mode, thermal ion diamagnetic drift and effective hot particle diamagnetic drift frequencies with the wave number $k_{\alpha}$ for $\left\langle\beta_{\text {dia }}\right\rangle=4.79 \%$ at $s=0.599$ on the field line that crosses the most destabilizing curvature region in LHD.

In figure 11 the ratio of mode frequency to the total drift frequency is plotted as a function of $s$, for $\left\langle\beta_{\mathrm{dia}}\right\rangle=4.79 \%$, $k_{\alpha}=10, N_{\mathrm{h} 0} / N_{\mathrm{i} 0}=0.01$ and 0.001 . For $k_{\alpha}=10$ the ordering $\omega / \omega_{\mathrm{dh}}$ is well satisfied for $N_{\mathrm{h} 0} / N_{\mathrm{i} 0}<0.01$; we estimate that it could still remain satisfactory up to 0.03 . The final figure (figure 12) summarizes all the important results. Total magnetic drift, mode, thermal ion diamagnetic drift and effective hot particle diamagnetic drift are plotted as a function of the wave number $k_{\alpha}$ for $\left\langle\beta_{\text {dia }}\right\rangle=4.79 \%$ at the most destabilized surface. $\omega_{* \text { heff }}$ is weak compared with $\omega_{* \mathrm{pi}}$ for large values of $k_{\alpha}$, but becomes comparable to it at $k_{\alpha} \sim 8$, and dominates for small $k_{\alpha}$ where ballooning theory breaks down. Similarly, $\omega$ and $\omega_{\mathrm{dh}}$ become comparable at $k_{\alpha} \sim 2$, but $\omega \gg \omega_{\mathrm{dh}}$ for $k_{\alpha}>8$. Once again it is visible here that for small $k_{\alpha}, \omega$ scales with $\omega_{* \text { heff }}$, for large $k_{\alpha}$, however, $\omega$ scales with $\omega_{* \mathrm{pi}}$.

\section{Summary and conclusion}

The ballooning stability can impose very strict operating constraints in current-free stellarators as the LHD and ideal 
MHD yield to inconclusive prediction about LHD stability for $\left\langle\beta_{\text {dia }}\right\rangle>3 \%$. We have applied a ballooning mode theory based on large hot particle drifts and finite diamagnetic drift corrections to a model LHD heliotron with $\left\langle\beta_{\text {dia }}\right\rangle$ up to $4.8 \%$, which is the actually achieved value in experiments. The ballooning theory that is applied is based on a kinetic ballooning mode equation where the hot particle drifts are ordered to be much larger than the typical mode frequency (or growth rate $\gamma_{\mathrm{F}}$ ). The MHD limit of this equation reduces then to a second order ordinary differential equation which can be solved with standard techniques in the fluid limit, where the diamagnetic drifts are ignored. A perturbative approach is considered to then examine the impact of the diamagnetic drift corrections by taking the fluid mode structure as a test eigenfunction to obtain a quadratic dispersion relation for the complex frequency of the mode. To determine whether this ordering $\omega / \omega_{\mathrm{dh}} \ll 1$ is satisfactory, we compute a mode-extent averaged contribution $\left\langle\omega_{\mathrm{dh}}\right\rangle$, since $\omega_{\mathrm{dh}}$ is a strongly oscillating function. That should have a weak effect where the fluid mode structure is nearly vanishing and be important where the mode becomes localized.

The fluid limit (with reduced hot particle pressure gradient drive and the absence of diamagnetic drifts) predicts a ballooning unstable band that moves from the plasma core, at low $\left\langle\beta_{\text {dia }}\right\rangle$, to the plasma boundary, at $\left\langle\beta_{\text {dia }}\right\rangle \approx 5 \%$, encompassing roughly $1 / 3$ of the plasma volume. When diamagnetic drifts are included, the ballooning mode theory predicts that the LHD device is stable to ballooning modes because $\left|\omega_{* \mathrm{pi}}+\omega_{* \text { heff }}\right| / 2>\gamma_{\mathrm{F}}$ for $0 \leqslant\left\langle\beta_{\text {dia }}\right\rangle \leqslant 4.8 \%$.

It has been verified that the ballooning mode frequency is effectively much smaller than the mode-width averaged hot particle (curvature and grad-B) drifts. Although this may not indisputably justify the expansion in $\omega / \omega_{\mathrm{dh}}$, it does represent a useful validation of the theory applied. We found that the effective hot particle diamagnetic drift has a weak impact on stability properties compared with the thermal ion diamagnetic drift. This is consistent with the fact that if the hot particle drifts (curvature and/or grad-B) are too fast to contribute significantly to the instability, they also must have a feeble impact on stability.

Finally all the results obtained suggest that driftmagnetohydrodynamics may constitute a more appropriate model for current-free stellarator/heliotron stability than ideal MHD or other enhanced (anisotropic) single-fluid approaches. A non-perturbative approach to deal with complex eigenvalue problem expressed in equation (1) constitutes an interesting topic of future research.

\section{Acknowledgments}

This work was supported in part by the Swiss National Science Foundation and by Euratom.

Euratom@2010.

\section{References}

[1] Weller A. et al 2009 Nucl.Fusion 49065016

[2] Yamaguchi T. et al 2005 Nucl. Fusion 45 L33

[3] Nakajima N. 1996 Phys. Plasmas 34556

[4] Watanabe K.Y. et al 2005 Nucl. Fusion 451247

[5] Kruskal M.D. and Oberman C.R. 1958 Phys. Fluids 1275

[6] Johnson J.C., Kulsrud R.M. and Weimer K.E. 1969 Plasma Phys. 11463

[7] Cooper W.A. et al 2006 Fusion Sci. Technol 50245

[8] Cooper W.A., Graves J.P., Jucker M., Watanabe K.Y., Narushima Y. and Yamaguchi T. 2007 Plasma Phys. Control. Fusion 491177

[9] Nakajima N., Hudson S.R. and Hegna C.C. 2007 Fusion Sci. Technol. $\mathbf{5 1} 79$

[10] Cooper W.A., Singleton D.B. and Dewar R.L. 1996 Phys. Plasmas 3275

[11] Cooper W.A. 1982 Plasma Phys. 24265

[12] Tang W.M., Dewar R.L. and Manickam J. 1982 Nucl. Fusion 221079

[13] Nakajima N., Hudson S.R., Hegna C.C. and Nakamura Y. 2006 Nucl. Fusion 46177

[14] Cooper W.A. 1983 Phys. Fluids 261830

[15] Cooper W.A. 2006 Proc. Joint Varenna-Lausanne Int. Workshop on Theory of Fusion Plasmas, Varenna, Italy (New York: AIP) p 300

[16] McMillan B.F. and Dewar R.L. 2006 Nucl. Fusion 46477

[17] Cooper G.A., Graves J.P., Cooper W.A., Gruber R. and Peterson R.S. 2009 J. Comput. Phys. 2284911

[18] Cooper W.A. et al 2009 Comput. Phys. Commun. 180 1524-33

[19] Cooper W.A. et al 2006 Nucl. Fusion 46 683-98

[20] Harvey R.W., McCoy M.G., Kerbel G.D. and Chiu S.C. 1986 Nucl. Fusion 2643

[21] McClements K.G., Dendy R.O., Hastie R.J. and Martin T.J. 1996 Phys. Plasmas 32994

[22] Catto P.J., Tang W.M. and Baldwin D.E. 1981 Plasma Phys. 23639

[23] Rewoldt G., Ku L.-P., Tang W.M. and Cooper W.A. 1999 Phys. Plasmas 64705

[24] Dewar R.L. and Glasser A.H. 1983 Phys. Fluids 26 3038-52

[25] Cooper W.A. 1992 Plasma Phys. Control. Fusion 341011 